\title{
PRODUCTIVIDAD Y CALIDAD NUTRICIONAL DE GENOTIPOS DE SORGO PARA DOBLE PROPÓSITO ${ }^{1}$
}

\author{
Nury Gutiérrez-Palacios ${ }^{2}$,Zildghean Chow-Wong ${ }^{3}$, Denis Bastianelli ${ }^{4}$, Laurent Bonnal ${ }^{4}$, Rafael Obando-Solís ${ }^{2}$, \\ Gilles Trouche
}

\section{RESUMEN}

Productividad y calidad nutricional de genotipos de sorgo para doble propósito. El objetivo del presente trabajo fue evaluar genotipos de sorgo para productividad de grano y rastrojo, así como la calidad nutricional para la alimentación del ganado. En los años 2003, 2004 y 2005 se evaluaron 26, 15 y 8 genotipos, respectivamente, con el testigo INTA CNIA en época de postrera (agosto-noviembre), en las instalaciones del Centro Nacional de Investigación Agropecuaria (INTACNIA), Nicaragua. Se midieron variables agronómicas y se determinó la calidad nutricional de hojas y tallos, usando el método de espectrometría en el infrarrojo cercano. Para todas las variables agronómicas y de calidad nutricional, se encontraron diferencias significativas entre genotipos, a excepción de rendimiento de grano en 2004 y contenido de fibra detergente neutro y ácido en tallos. La heredabilidad de características agronómicas y de los componentes de calidad en hojas fue alta $(\geq 0,50)$, con valores comprendidos entre 0,96 y 0,51 e inferior para la mayoría de los parámetros de calidad en tallos, comprendidos entre 0,47 y 0,20. La única correlación positiva fue entre materia proteica total (MAT) y hoja/rastrojo fresco (H/RF) en los tallos. Los genotipos BF 95-11/195, Sureño, SH 688, V 142 y V 144 sobresalieron en rendimiento de grano, rastrojo y calidad nutricional (hojas-tallos). Sureño superó en materia proteica bruta por hectárea (MPB/ha) al testigo en 50 y $33 \%$ en hojas y tallos, respectivamente.

Palabras claves: ganado, digestibilidad, variabilidad genética.

\begin{abstract}
Productivity and nutritional quality of double purpose sorghum genotypes. The objective of this study was to evaluate a set of dual purpose sorghum genotypes for their grain and fodder productivity, as well as for nutritional quality of leaves and stems for livestock. From 2003 through 2005, 26, 15 and 8 genotypes were evaluated respectively, in comparison with the control variety INTA CNIA during the postrera season (August to November) in the experimental fields of the National Agricultural Research Centre (INTACNIA), Nicaragua. Agronomic traits were measured as well as nutritional quality of leaves and stems using the Near Infrared Spectrometry method. For all agronomic and nutritional quality traits were found significant differences among genotypes, except for grain yield in 2004, and the neutral and acid detergent fiber contents in stems. The heritability of agronomic and quality traits in leaves was high $(\geq 0.50)$, with values between 0.96 and 0.51 but lower for most of quality parameters in stems, with values between 0.47 and 0.20 . The only significant positive correlation was among crude protein (MAT) and leave - stem ratio in fresh stems (H/RF). The genotypes BF 95-11/195, Sureño, SH 688, V142 and V 144, achieved the best results for grain and fodder yield and fodder quality (regarding both leaves and stems). Sureño out-yielded the control variety for crude protein content per hectare (MPB/ha) in 50 and $33 \%$ for leaves and stems respectively.
\end{abstract}

Key words: livestock, digestibility, genetic variability.

1 Recibido: 15 de febrero, 2012. Aceptado: 1 de abril, 2013. Proyecto de investigacion. Instituto Nicaragüense de Tecnología Agropecuaria (INTA), Nicaragua.

2 Instituto Nacional de Tecnología Agropecuaria INTA, Contiguo a Estación 5 de Policía, Managua, Nicaragua. nury.gutierrez@yahoo.es, raobando@inta.gob.ni

3 Centro Internacional de Agricultura Tropical (CIAT), Restaurante Marsellaise 2 c lago, casa 303. Apartado postal LM-172, Managua, Nicaragua.z.g.chow@cgiar.org

4 Centre de Cooperation Internationale en Recherche Agronomique Pour le Développement CIRAD-SELMET, Campus de Baillarguet F34398 Montpellier cedex 5, Francia. denis.bastianelli@cirad.fr, laurent.bonnal@cirad.fr

5 CIRAD-Aiva, Avenue Agropolis, F34398 Montpellier, Francia. gilles.trouche@cirad.fr 


\section{INTRODUCCIÓN}

El sorgo [Sorghum bicolor (L) Moench], es el quinto cereal más importante a nivel mundial después del trigo, maíz, arroz y cebada (Dahlberg et al. 2011), es un grano básico importante para las zonas tropicales semiáridas en muchos países de África, Asia y América Central, siendo uno de los principales componentes de la dieta alimenticia para millones de habitantes pobres del mundo (FAO 1995).

En los trópicos semiáridos, el sorgo se utiliza principalmente como cultivo de doble propósito, produciendo grano para el consumo humano y rastrojo para la alimentación de ganado (Bramel-Cox et al. 1995). En África el rastrojo de sorgo es de considerable importancia económica, ya que se utiliza como forraje para la ganadería extensiva (Youngquist et al. 1990). El ganado obtiene el $45 \%$ de su alimento de los residuos de cosechas en varias partes de SubSahara África y el $80 \%$ en períodos críticos de sequía (Sanford 1989). En las áreas semiáridas de Asia, en particular en la India, el rastrojo del sorgo es también usado extensivamente para alimento del ganado (Kelly y Rao 1993). En la India, alrededor del 50 a 60\% del total de los alimentos es obtenido de los residuos de cosecha (Parthasarathy y Hall 2003).

En Nicaragua, en el ciclo agrícola 2008-2009, el sorgo ocupó el 5,96\% del área sembrada de granos básicos con un rendimiento de 2,1 t/ha (MAGFOR 2009). Esta producción se obtiene en una gran diversidad de sistemas productivos y explotaciones agrícolas incluyendo las fincas ganaderas. El mayor porcentaje de uso del cultivo es de doble propósito utilizando el rastrojo para alimentar al ganado durante la época seca, en forma de pastoreo después de la cosecha del grano o en la elaboración de pacas para alimentación en verano (Pastora y Téllez 2004).

En Nicaragua, el rastrojo o guate de sorgo es utilizada como forraje que se suministra a los animales, entre los meses de febrero y mayo (época seca), generalmente sin ningún aditivo, mientras se recuperan los pastizales (Trouché et al. 2006). Son pocos los agricultores que pican el rastrojo en trozos pequeños, ya que generalmente los animales se alimentan mediante el pastoreo. En las comunidades con mejor comunicación vial, los productores elaboran pequeños manojos de rastrojo de cinco libras que luego son vendidos a los ganaderos en la época seca.
El rastrojo de sorgo constituye una excelente fuente de fibra (8 a 10 toneladas de forraje por hectárea), disponible en un momento "clave" en el manejo de pasturas y animales. El rastrojo es normalmente de baja calidad, en las condiciones de pastoreo que normalmente son utilizados, presentando una baja ganancia diaria debido a que este tipo de forraje se degrada muy lentamente en rumen, ocasionando una disminución en el consumo voluntario de materia seca atribuible a una baja tasa de pesaje (Mieres et al. 2011).

Un factor limitante en el uso del sorgo para doble propósito es la calidad nutricional de los rastrojos ya que la energía digestible, proteína cruda y minerales son bajos (Bartle y Klopfenstein 1988). Ante esta problemática, los fitogenetistas de sorgo y mijo están claros de la importancia de mejorar no sólo el rendimiento en grano, sino también la cantidad y calidad del rastrojo. Un incremento de $1 \%$ en la digestibilidad del rastrojo del sorgo (Kristjanson y Zerbini 1999) podría inducir un incremento de 5\% en la producción de leche en ganado vacuno.

Diferentes estudios han reportado que existe una variabilidad genética en los contenidos de fibra y digestibilidad del rastrojo de sorgo, ambos en los estados de floración (Gupta et al. 1976, Lodhi y Dangi 1981, Caravetta et al. 1990) y cosecha (Ross et al. 1983, Youngquist et al. 1990, Badve et al. 1994).

Es posible que la variabilidad genética en la calidad del rastrojo pueda ser explotada para desarrollar el mejoramiento de germoplasmas con alto valor nutritivo del rastrojo (ILRI 2012).

El sorgo de doble propósito es una alternativa para mejorar los ingresos de las familias campesinas nicaragüenses mediante el uso del rastrojo de calidad para su ganado vacuno o la venta, sin afectar su seguridad alimentaria. El objetivo del presente trabajo fue evaluar genotipos de sorgo para productividad de grano y rastrojo, así como la calidad nutricional para la alimentación del ganado.

\section{MATERIALES Y MÉTODOS}

Los experimentos en campo se realizaron en los ciclos agrícolas 2003, 2004 y 2005, en época postrera (agosto - diciembre), en el Centro Nacional de Investigación Agropecuaria (CNIA), del Instituto Nicaragüense de Tecnología Agropecuaria (INTA), ubicado 
en el departamento de Managua, Nicaragua, a $12^{\circ} 08^{\prime}$ LN y $86^{\circ} 10^{\prime} \mathrm{LO}$, a una altitud de $56 \mathrm{msnm}$. Los suelos pertenecen al orden Andosol, serie sabana grande, con textura franco-arenoso (MAG 1971). Las precipitaciones y temperaturas promedios anuales son de 1229,6 $\mathrm{mm}$ y $27,5^{\circ} \mathrm{C}$, respectivamente (INETER 2004).

Los genotipos en estudio son variedades de polinización libre procedentes del CIRAD, ICRISAT e INTA (Cuadro 1). En los ciclos agrícolas 2003, 2004 y 2005 se evaluaron 26,15 y 8 genotipos, respectivamente. El diseño experimental utilizado fue de bloques completos al azar (BCA) con tres repeticiones. En cada ciclo se conservaron los mejores materiales con base en los criterios definidos para doble propósito. En 2004, se evaluaron los diez mejores genotipos, además cinco seleccionados en otros experimentos. La parcela experimental estuvo conformada por tres a seis surcos de $5 \mathrm{~m}$ de longitud y a una distancia entre surco de 0,75 metros.

La siembra se realizó manualmente, utilizando una densidad de $12,9 \mathrm{~kg} / \mathrm{ha}$ de semilla, se aplicó 128 $\mathrm{kg} /$ ha de fertilizante completo, mezclado con terbufós $(13 \mathrm{~kg} / \mathrm{ha})$ para control de plagas de suelo. A los quince días después de la emergencia, se realizó el raleo a dieciocho plantas por metro lineal para una población de 240000 plantas por hectárea. La fertilización nitrogenada con urea se aplicó a las tres y seis semanas después de la emergencia a una dosis de $64 \mathrm{~kg} / \mathrm{ha}$. El control de malezas se realizó manualmente a los 15 y 40 días después de la emergencia.

Las variables agronómicas evaluadas fueron las siguientes: días a floración (DAF), altura de planta (APL), grados Brix (10-12 días después del 50\% de floración, tomando un entrenudo en la parte media del tallo). Al momento de la madurez fisiológica se cosecharon los dos surcos centrales de cada parcela experimental. Se tomó el peso de panojas (PPAN), y la humedad de una muestra de granos (HGR) para determinar su rendimiento al 14\% de humedad (RGR). Se pesó el rastrojo fresco de los mismos dos surcos; y de una muestra de seis plantas, se separó hojas y tallos

Cuadro 1. Genotipos de sorgo de doble propósito evaluados en los años 2003, 2004 y 2005. Centro Nacional de Investigación Agropecuaria (CNIA), Managua, Nicaragua.

\begin{tabular}{lll}
\hline $\mathbf{2 0 0 3}$ & \multicolumn{1}{c}{$\mathbf{2 0 0 4}$} & \multicolumn{1}{c}{ 2005 } \\
\hline ICSR 9 & BF 95-11/110 & BF 94-6/46K-1K-1K-1F \\
ICSR 20 & BF 95-11/195 & BF 96-2/46-1K-1K-1K \\
ICSR 26 & BF 95-11/160 & V 144 \\
ICSR 29 & BF 93-24/51-2-1 & ICSR 29 \\
ICSR 41 & BF 94-6/46K-1K-1K-1F & ICSR 89064 \\
ICSR 101 & BF 96-2/46-1K-1K-1K & Africana \\
ICSR 161 & V 142 & Sureño \\
ICSR 98048 & V 144 & INTA CNIA \\
ICSR 89064 & V 161 & \\
ICSR 90005 & ICSR 29 & \\
ICSR 90001 & ICSR 161 & \\
SH 910 & ICSR 89064 & \\
SH 688 & SH 688 & \\
IS 21891 & Sureño & \\
BF 93-24/51-2-1 & INTA CNIA & \\
V 25 & & \\
V 52 & & \\
V 142 & & \\
V 144 & & \\
V 156 & & \\
V 161 & & \\
IS 13868 & & \\
Real 70 & & \\
Pinolero 1 & & \\
Sureño & & \\
INTA CNIA & & \\
\hline
\end{tabular}


para medir el peso húmedo, y el contenido de materia seca se determinó después de secar una muestra en el horno a $65^{\circ} \mathrm{C}$ durante 72 horas. Cada muestra seca se molió y se tamizó $(1 \mathrm{~mm})$ para los análisis de laboratorio. En base a estos resultados se calculó las siguientes variables: rendimiento fresco de rastrojo (RFR), rendimiento seco de rastrojo (RSR), rendimiento seco de tallos (RST), rendimiento seco de hojas (RSH), relación hoja/rastrojo seco (H/RS) medida en porcentaje de materia seca y hoja/rastrojo fresco (H/RF).

Los análisis bioquímicos de las muestras de las hojas (2003) y tallos (2004) se realizaron en el laboratorio de nutrición animal de la unidad SELMET del CIRAD en Montpellier (Francia). Las variables medidas fueron: minerales totales (MM), materia proteica total (MAT, método Kjeldahl), fibra evaluada por el método de Van Soest (Van Soest y Wine 1967): fibra detergente neutro (NDF), fibra detergente ácido (ADF), lignina ácido detergente (ADL), digestibilidad enzimática in vitro (pepsina + celulasa) de la materia seca (SMS), todas estas medidas expresadas en porcentaje de materia seca (Bastianelli y Hervouet 2000). Con base en estos parámetros estándar, se calculó dos parámetros compuestos: ADL/NDF que representa la proporción de ligninas en fibras totales y digestibilidad de NDF (IVNDFD), que estima la digestibilidad específica de las fibras constituyentes de las paredes celulares, calculado con la fórmula (Barriere et al. 2008):

$$
\operatorname{IVNDFD}=100 *((\mathrm{SMS}-(100-\mathrm{NDF})) / \mathrm{NDF}
$$

La determinación de la composición química fue realizada por espectrometría en el infrarrojo cercano (NIRS, Near InfraRed Spectroscopy). Los espectros fueron tomados con un espectrómetro NIRSYSTEM 6500 (FOSS, Laurel (MD), USA) en modo de reflectancia, a longitudes de onda entre 400 y $2500 \mathrm{~nm}$. Se utilizaron copelas provistas de cuarzo para presentar las muestras finamente molidas de hojas y tallos. La predicción fue realizada con base en ecuaciones de calibración para el rastrojo de sorgo existente en el CIRAD, utilizando ocho muestras para ajustar las ecuaciones de predicción NIRS. Estas ecuaciones fueron ajustadas a las muestras del estudio por medio de análisis de referencia de laboratorio (método estándar del AOAC de ocho muestras seleccionadas por presentar el espectro de diversidad). El método NIRS es una herramienta eficaz y precisa para estimar la composición bioquímica del rastrojo de sorgo.
Un parámetro de estimación del valor nutricional del rastrojo fue calculado en base a la composición química, utilizando ecuaciones definidas por el INRA (Colin-Schoellen et al. 2000): digestibilidad in vivo (estimada) de la materia orgánica (dMO), expresada en $\%$ de materia seca y en $\mathrm{kg} / \mathrm{ha}$. La materia proteica total (MAT) fue también expresada en $\mathrm{kg} / \mathrm{ha}$ (MPB). Para $\mathrm{dMO} /$ ha en tallos y MPB/ha en hojas y tallos, se utilizaron las fórmulas siguientes:

$\mathrm{dMO}$ t/ha $=\mathrm{dMOt} * \mathrm{RTO} \mathrm{MO}$, donde: $\mathrm{dMOt}=$ es el coeficiente de $\mathrm{dMO}$ en porcentaje de materia seca en los tallos; RTO MO= rendimiento de la materia orgánica

RTO MO = RTO MS * (1-MM/100), donde: RTO $\mathrm{MS}=$ es el rendimiento de la materia seca de los tallos

$\mathrm{MPB} / \mathrm{ha}=\mathrm{RST} * \mathrm{MAT} * 10$, donde: $\mathrm{RST}=$ es el rendimiento seco de tallos y en el caso de las hojas (RSH)

Para los análisis estadísticos, se utilizó el programa XLSTAT 2009.6.02, realizando los análisis de varianza (ANOVA) de todas las variables agronómicas y calidad del rastrojo, las comparaciones múltiples de medias (prueba LSD Fisher con una probabilidad de $\mathrm{P} \leq 0,05$ ), $\mathrm{y}$ análisis de componentes principales (ACP) para estudiar la correlación entre variables agronómicas y calidad de hojas y tallos.

La heredabilidad en sentido amplio $\left(\mathrm{H}^{2}\right)$, se estimó de acuerdo a los resultados del ANOVA, utilizando las siguientes fórmulas:

$$
\begin{aligned}
& \mathrm{V}_{\mathrm{G}}=\left(\mathrm{CM}_{\mathrm{G}}-\mathrm{CM}_{\mathrm{E}}\right) / \mathrm{k} \\
& \mathrm{V}_{\mathrm{A}}=\mathrm{CM}_{\mathrm{E}} \\
& \mathrm{h}^{2}=\mathrm{V}_{\mathrm{G}} /\left(\mathrm{V}_{\mathrm{G}}+\mathrm{V}_{\mathrm{A}}\right)
\end{aligned}
$$

donde: $\mathrm{V}_{\mathrm{G}}=$ varianza genética; $\mathrm{CM}_{\mathrm{G}}=$ cuadrado medio del factor genotipos; $\mathrm{CM}_{\mathrm{E}}=$ cuadrado medio del error; $\mathrm{k}=$ número de repeticiones del experimento; $\mathrm{V}_{\mathrm{A}}=$ varianza ambiental; $\mathrm{h}^{2}=$ heredabilidad.

\section{RESULTADOS Y DISCUSIÓN}

\section{Ecuaciones de calibración NIRS de los parámetros de calidad}

La precisión de la predicción de los parámetros analíticos fue satisfactoria y próxima a los datos 
clásicamente obtenidos para este tipo de parámetros/ plantas (Murray et al. 2008). Los coeficientes de determinación fueron superiores a 0,95 , a excepción de ADL en tallos $(0,89)$. La precisión en SMS es importante, ya que es una medida de degradación enzimática, por lo tanto, a priori menos fácil a predecir según el espectro infrarrojo (datos no presentados). Los datos disponibles de este estudio son precisos y podrían ser utilizados en trabajos de fitomejoramiento.

\section{Características agronómicas y calidad nutricional de hojas y tallos de los genotipos estudiados}

Las precipitaciones registradas en el CNIA en el 2003, 2004 y 2005 durante el período de evaluación en campo (agosto-diciembre) fueron de 534, 396 y $749 \mathrm{~mm}$, respectivamente (datos no presentados). En 2005, las condiciones climáticas favorecieron a que los genotipos expresaran su potencial de rendimiento, contrario al 2004.

\section{Variables agronómicas}

Se observaron diferencias altamente significativas y significativas entre los genotipos para todas las variables medidas, a excepción del RGR en el 2004. En general, los coeficientes de variación fueron inferiores al $20 \%$, indicando precisión y confiabilidad aceptable de los datos medidos, excepto RFR, RSH, H/RF y H/ RS. Todas las variables analizadas en las hojas mostraron una heredabilidad superior a 0,5; sin embargo, en el caso de los tallos sólo en el contenido de grados Brix, RFR, RSR y RST; indicando la posibilidad de utilizar esta información en programas de selección y mejoramiento. En los dos años de estudio, DAF y APL alcanzaron una heredabilidad superior a 0,90. En 2004, el RGR presentó una heredabilidad muy baja $(0,05)$ debido a la sequía y problemas de acame temprano en algunos genotipos. Esta también afectó el RFR al reducir la altura de planta y el rendimiento de tallos (Cuadro 2).

Cuadro 2. Resultados principales de los análisis de varianza (ANOVA) para las variables agronómicas y calidad nutricional de hojas y tallos medidos en los genotipos de sorgo de doble propósito. Centro Nacional de Investigación Agropecuaria (CNIA), Managua, Nicaragua. 2003 y 2004.

\begin{tabular}{|c|c|c|c|c|c|c|c|c|c|}
\hline \multirow[b]{2}{*}{ Variables } & \multicolumn{4}{|c|}{ Ensayo de 26 líneas (2003) } & \multicolumn{5}{|c|}{ Ensayo de 15 líneas (2004) } \\
\hline & Media & Fc & CV (\%) & $\mathbf{h}^{2}$ & Variables & Media & Fc & CV (\%) & $\mathbf{h}^{2}$ \\
\hline DAF & 67 & $* * *$ & 1,9 & 0,93 & DAF & 60 & $* * *$ & 1,2 & 0,97 \\
\hline APL & 210 & $* * *$ & 4,5 & 0,96 & APL & 208 & $* * *$ & 4,3 & 0,96 \\
\hline BRIX & 6,34 & $* * *$ & 17,2 & 0,76 & BRIX & 7,27 & $* * *$ & 16,9 & 0,56 \\
\hline RGR & 4454 & $* * *$ & 17,5 & 0,62 & RGR & 5918 & NS & 16,1 & 0,05 \\
\hline RFR & 27,18 & $* * *$ & 23,6 & 0,68 & RFR & 24,48 & $* * *$ & 15,3 & 0,56 \\
\hline RSH & 1,88 & $* * *$ & 30,1 & 0,51 & RST & 5,01 & $* * *$ & 16,9 & 0,72 \\
\hline $\mathrm{H} / \mathrm{RF}$ & 12,19 & $* *$ & 20,8 & 0,67 & $\mathrm{H} / \mathrm{RS}$ & 28,00 & $* *$ & 25,6 & 0,35 \\
\hline MM-hojas & 13,72 & $* * *$ & 6,3 & 0,75 & $\mathrm{H} / \mathrm{RF}$ & 16,06 & $*$ & 28,9 & 0,31 \\
\hline MAT-hojas & 7,67 & $* * *$ & 7,9 & 0,67 & RSR & 6,85 & $* * *$ & 13,0 & 0,69 \\
\hline NDF-hojas & 62,97 & $* * *$ & 1,8 & 0,82 & MM-tallos & 9,77 & $*$ & 13,4 & 0,30 \\
\hline ADF-hojas & 33,34 & $* * *$ & 2,5 & 0,86 & MAT-tallos & 2,82 & $* * *$ & 20,0 & 0,38 \\
\hline ADL-hojas & 3,42 & $* * *$ & 5,8 & 0,77 & NDF-tallos & 66,60 & NS & 6,0 & 0,24 \\
\hline \multirow[t]{6}{*}{ SMS-hojas } & 46,42 & $* * *$ & 2,3 & 0,72 & ADF-tallos & 41,76 & NS & 7,2 & 0,20 \\
\hline & & & & & ADL-tallos & 5,37 & $* *$ & 11,7 & 0,32 \\
\hline & & & & & SMS-tallos & 38,27 & $*$ & 10,3 & 0,26 \\
\hline & & & & & dMO-tallos & 51,17 & $*$ & 5,1 & 0,26 \\
\hline & & & & & ADL/NDF & 8,08 & $* * *$ & 10,3 & 0,44 \\
\hline & & & & & IVNDFD & 7,31 & $* * *$ & 25,2 & 0,47 \\
\hline
\end{tabular}

$*, * *, * * *$ significativo al 5,1 y $0,1 \%$, respectivamente.

Fc: probabilidad según Tukey, NS: no significativo, CV: coeficiente de variación, $\mathrm{h}^{2}$ : heredabilidad. 
Los rendimientos de grano promedio obtenidos en los dos años fueron superiores a los encontrados en otras evaluaciones de sorgo de doble propósito. García y García (2007) reportaron rendimiento de grano en genotipos de doble propósito de $4000 \mathrm{~kg} / \mathrm{ha}$ con aplicaciones de niveles de nitrógeno; $3179 \mathrm{~kg} / \mathrm{ha}$ (Hemanth et al. 1998) y $2200 \mathrm{~kg} / \mathrm{ha}$ (Rattunde 1998). Este resultado se explica por la excelente fertilidad de los suelos del CNIA, y por condiciones favorables como temperaturas elevadas, alta radiación solar, baja incidencia de enfermedades y un buen manejo del cultivo.

En los dos años, el RFR varió de 17,7 a 55,5 t/ ha, estos resultados son similares a los reportados por García (1981), Corrales (1986), Villegas (1990) y Amador y Boschini (2000). Una relación alta de peso de las hojas en rastrojo resulta en una mayor digestibilidad y viceversa (Fales 1986). Se ha evaluado la relación hoja-tallo, para explicar la calidad del rastrojo (Vogel et al. 1981). En nuestros resultados la relación $\mathrm{H} / \mathrm{RF}$ en hojas fue de 12,2 y en tallos de 16,1 (Cuadro 2).

\section{Variables de calidad nutricional en hojas y tallos}

Los resultados obtenidos en los parámetros de calidad en hojas y tallos muestran diferencias significativas entre los genotipos, excepto en NDF y ADF en tallos, coincidiendo con lo observado por Vargas (2005).

El coeficiente de variación para los parámetros de calidad en hojas estuvo comprendido entre 1,9 y $8,0 \%$ y presentaron una alta heredabilidad $(0,67-0,86)$. En tallos, el coeficiente de variación fue mayor $(5,1$ a $20,0 \%)$ y la heredabilidad baja $(0,20-0,47)$ para la mayoría de los parámetros de calidad.

Estudios con forrajes sugieren que la calidad puede ser incrementada simultáneamente con la producción (Zerbini y Thomas 2003). La heredabilidad alta observada en los parámetros de calidad de las hojas debe ser utilizada en los programas de selección y mejorar la calidad nutricional de esta parte de la planta. Además, la heredabilidad permitiría evaluar las ventajas de los diferentes métodos de mejoramiento (Hanson 1963). A diferencia en los tallos esta fue baja, en este caso, demandarán de métodos más elaborados para mejorar determinados caracteres (Resende 2002). Estos resultados coinciden con los encontrados en este estudio.
Los valores promedios de MM, MAT y SMS fueron superiores en las hojas, y los contenidos de NDF, ADF y ADL mayores en los tallos (Cuadro 2), indicando mejor calidad en las hojas; estos resultados coinciden con los de White et al. (1981). Boschini y Elizondo (2005), encontraron contenidos menores de MM en hojas y en tallos; de igual manera McDowell (1974) y Vargas (2005) reportaron valores menores en hojas. En sorgos forrajeros, Boschini y Amador (2000) y Boschini y Elizondo (2005) determinaron valores superiores de MAT en hojas y tallos, con 15,98 y $5,24 \%$, y 13,51 y $4,83 \%$, respectivamente.

Los contenidos de NDF en hojas y tallos fueron entre $58,1-64,0 \%$ y $60,9-72,7 \%$, respectivamente (Cuadro 2); resultados similares a los encontrados por Amador y Boschini (2000), Rattunde et al. (2001) y Bolletta y Vallati (2006), en un estudio de seis cultivares de sorgo de doble propósito, señalaron que el contenido de NDF en tallos favorece el mejoramiento de esta variable en relación a las hojas.

El valor promedio de ADF en hoja y tallos fue superior al encontrado por Amador y Boschini (2000), sin diferir al reportado por Vargas (2005), representando valores de calidad importante en la digestibilidad de rumiantes.

La lignificación de las paredes celulares de las plantas ha sido considerada como el principal factor que limita la digestibilidad de los forrajes. Se encontraron contenidos de ADL en los tallos (5,37\%) y hojas $(3,42 \%)$, similares $(4,7 \%$ en tallos y hojas) a los reportados por Bolleta y Vallati (2006).

\section{Variabilidad de los componentes de calidad en ho- jas y tallos}

Es importante señalar que los análisis se realizaron en diferentes años para los parámetros en hoja (2003) y en tallos (2004), y no se puede negar un efecto de las condiciones climáticas. La desviación estándar fue mayor en los tallos que en las hojas para las variables relacionadas con los constituyentes de la fibra y la digestibilidad, coincidiendo con lo reportado por Rattunde et al. (2001). Esto favorece la selección de los parámetros de calidad en los tallos, mientras que en los contenidos de MM y MAT se observaron valores de desviación estándar mayores en las hojas (Figura 1). 


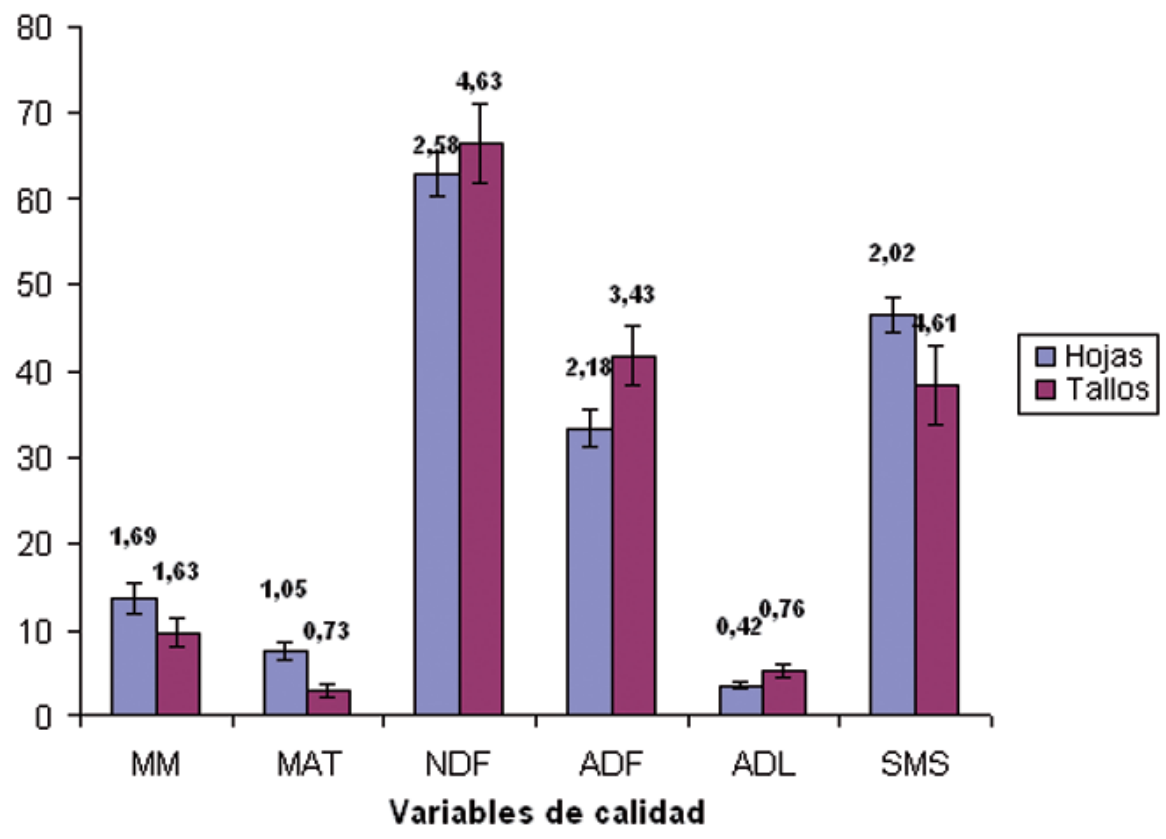

Figura 1. Promedios y desviación estándar de los componentes de calidad nutricional en hojas y tallos de genotipos de sorgos doble propósito. Centro Nacional de Investigación Agropecuaria (CNIA), Managua, Nicaragua. 2003-2004.

MM: minerales totales, MAT: materia proteica total, NDF: fibra neutro detergente, ADF: fibra ácido detergente, ADL: lignina ácido detergente, SMS: digestibilidad de la materia seca.

\section{Relación entre las variables agronómicas y valor nutricional del rastrojo}

Las correlaciones entre las variables agronómicas y calidad nutricional del rastrojo medidos en los 26 y 15 genotipos evaluados en 2003 y 2004, respectivamente, se resumen en las gráficas de los ACP (Figura 2 y 3 ).

En el experimento del 2003, se observa que el plano factorial definido por los dos primeros componentes principales (CP) explica el $62,4 \%$ de la variabilidad total en el conjunto de las variables agronómicas y variables de calidad nutritiva de hojas. El CP-1, explica el $38,2 \%$ de la variabilidad total, el contenido de NDF, ADF y ADL presentan una alta correlación positiva entre sí y negativa con las variables MAT, BRIX y SMS, igualmente relacionadas entre ellas. El
CP-2 está definido por las variables de RGR, RFR, RSH y APL, las tres últimas con una alta correlación positiva (Figura 2).

En el experimento del 2004, se observa que el plano factorial definido entre los dos primeros $\mathrm{CP}$, explica el $72,1 \%$ de la variabilidad total en el conjunto de las variables agronómicas y variables de calidad nutritiva de tallos. El CP-1, explica el $48,6 \%$ de la variabilidad total. El contenido de ADL mostró correlaciones negativas y significativas con $\mathrm{H} / \mathrm{RF}$, pero positivas con RSR y RST, esto indica la mayor concentración de ligninas en los tallos que es el factor limitante en la digestibilidad de los forrajes (Nuñez y Cantú 2001). El parámetro H/RF podría ser un elemento principal en la calidad de la planta al mostrar una correlación positiva con MAT, 


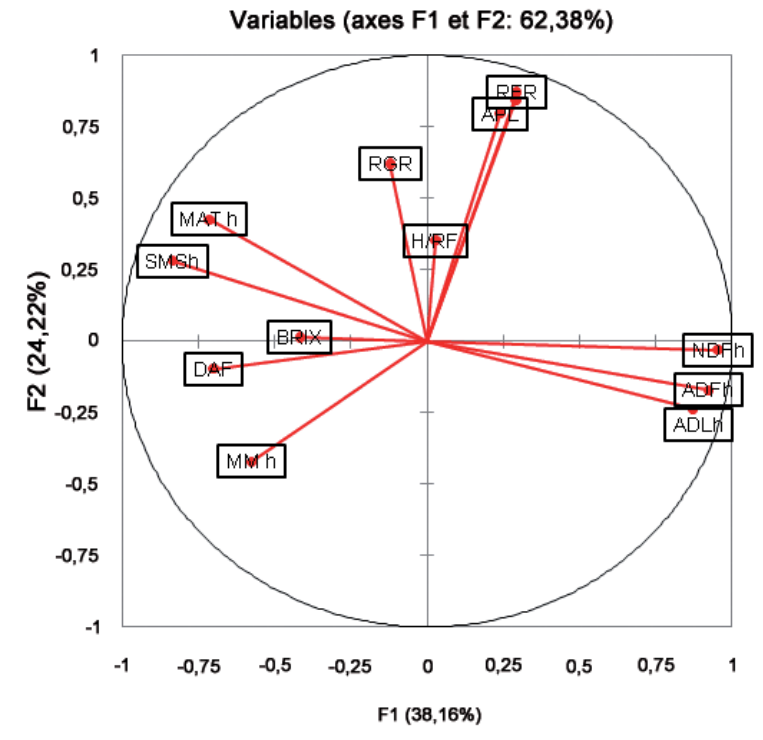

Figura 2. Análisis de los componentes principales (ACP) de las variables agronómicas y calidad nutricional de hojas medidas en 26 genotipos de sorgo de doble propósito. Centro Nacional de Investigación Agropecuaria (CNIA). Managua, Nicaragua. 2003.

DAF: días a 50\% de floración, RGR: rendimiento de grano, RFR: rendimiento fresco de rastrojo, RSH: rendimiento seco de hojas, H/RF: relación hoja rastrojo fresco, APL: altura de planta, BRIX: grados Brix, MAT: materia proteica total, MM: minerales totales, NDF: fibra detergente neutro, ADF: fibra detergente ácido, ADL: lignina ácido detergente, SMS: digestibilidad enzimatica in vitro de la materia seca. La letra h que sigue el nombre de las variables de calidad nutricional estudiadas se refiere a hojas.

Nota: En la gráfica RFR está confundida con RSH.

sugiriendo la importancia de hacer mejoramiento en esta variable.

El CP-2 está definido por las variables NDF y ADF, estas presentaron correlación positiva entre sí, y negativa con las variables $\mathrm{dMO}$ y SMS, igualmente relacionadas. Estas últimas no demostraron correlación alguna con las variables agronómicas. El RGR se correlacionó positivamente con MM, y no se observó correlación con las otras variables de calidad (Figura 3).

En el presente estudio no se encontraron correlaciones significativas entre los constituyentes de

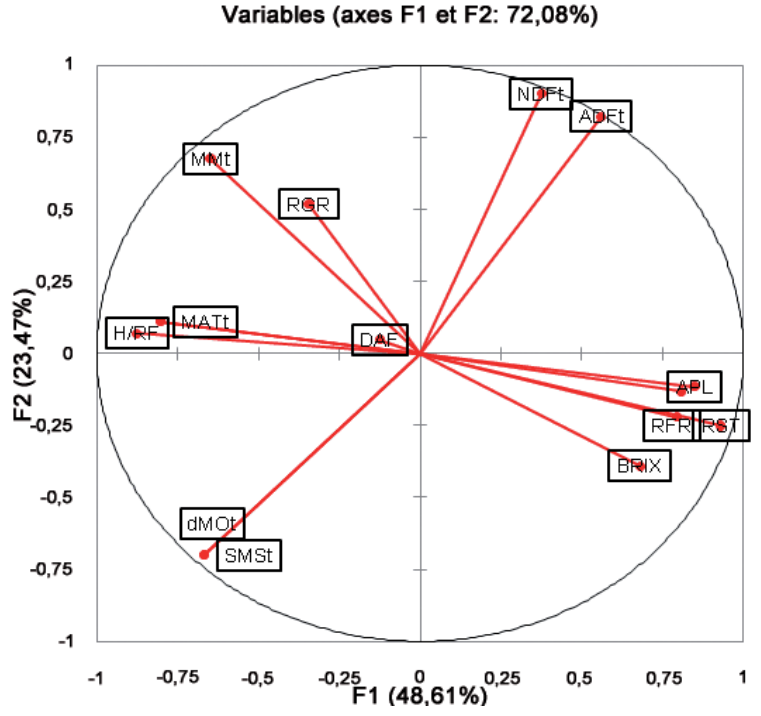

Figura 3. Análisis de los componentes principales (ACP) de variables agronómicas y calidad nutricional de tallos medidas en quince genotipos de sorgo de doble propósito. Centro Nacional de Investigación Agropecuaria (CNIA), Managua, Nicaragua, 2004.

DAF: días a $50 \%$ de floración, RGR: rendimiento de grano, H/ RF: relación hoja rastrojo fresco, APL: altura de planta, RFR: rendimiento fresco de rastrojo, RST: rendimiento seco de tallos, BRIX: grados Brix, MAT: materia proteica total, MM: minerales totales, NDF: fibra detergente neutro, ADF: fibra detergente ácido, ADL: lignina ácido detergente, dMO: digestibilidad in vivo de la materia organica, SMS: digestibilidad enzimatica in vitro de la materia seca. La letra $t$ que sigue el nombre de las variables de calidad nutricional estudiadas se refiere a tallos.

Nota: En la gráfica ADL está casi confundido con APL.

la fibra y los componentes de productividad (hojas y tallos), condición que permite seleccionar materiales que combinen alta productividad y calidad nutritiva. Una relación negativa entre calidad de forraje y rendimiento de forraje puede ser suficientemente débil, pero que de todas maneras permite el progreso (Shenk y Elliot 1971). Varios estudios mostraron que Una relación débil entre el rendimiento de grano y las características de calidad en residuos de cosecha, permite desarrollar variedades mejorando los valores del rastrojo sin sacrificar la producción de grano particularmente en sistemas de producción de bajos 
Cuadro 3. Calidad nutricional (hojas) de los mejores genotipos de sorgo de doble propósito. Centro Nacional de Investigación Agropecuaria (CNIA), Managua, Nicaragua. 2003.

\begin{tabular}{|c|c|c|c|c|c|c|c|}
\hline Variedades & RSH (t/ha) & RFR (t/ha) & MAT & NDF & SMS & ADL/NDF & MPB h $(\mathrm{kg} / \mathrm{ha})$ \\
\hline Sureño & $\begin{array}{c}3,66 \\
(181)\end{array}$ & $\begin{array}{l}55,48 \\
(260)\end{array}$ & $\begin{array}{l}7,07 \\
(82)\end{array}$ & $\begin{array}{c}64,0 \\
(103)\end{array}$ & $\begin{array}{c}47,31 \\
(99)\end{array}$ & $\begin{array}{c}6,03 \\
(116)\end{array}$ & $\begin{array}{c}259 \\
(150)\end{array}$ \\
\hline V144 & $\begin{array}{l}1,56 \\
(77)\end{array}$ & $\begin{array}{l}29,40 \\
(138)\end{array}$ & $\begin{array}{c}9,93 \\
(116)\end{array}$ & $\begin{array}{c}58,14 \\
(93)\end{array}$ & $\begin{array}{l}50,46 \\
(106)\end{array}$ & $\begin{array}{l}4,33 \\
(83)\end{array}$ & $\begin{array}{l}155 \\
(90)\end{array}$ \\
\hline V142 & $\begin{array}{c}2,24 \\
(111)\end{array}$ & $\begin{array}{l}37,16 \\
(174)\end{array}$ & $\begin{array}{c}8,87 \\
(104)\end{array}$ & $\begin{array}{c}61,61 \\
(99)\end{array}$ & $\begin{array}{l}49,87 \\
(105)\end{array}$ & $\begin{array}{l}4,72 \\
(91)\end{array}$ & $\begin{array}{c}199 \\
(115)\end{array}$ \\
\hline SH 688 & $\begin{array}{c}3,0 \\
(149)\end{array}$ & $\begin{array}{l}48,71 \\
(228)\end{array}$ & $\begin{array}{l}7,52 \\
(88)\end{array}$ & $\begin{array}{l}62,88 \\
(101)\end{array}$ & $\begin{array}{c}45,12 \\
(95)\end{array}$ & $\begin{array}{c}6,01 \\
(115)\end{array}$ & $\begin{array}{c}226 \\
(131)\end{array}$ \\
\hline ICSR 89064 & $\begin{array}{l}1,29 \\
(63)\end{array}$ & $\begin{array}{c}17,73 \\
(83)\end{array}$ & $\begin{array}{c}8,65 \\
(101)\end{array}$ & $\begin{array}{c}58,44 \\
(94)\end{array}$ & $\begin{array}{l}48,81 \\
(102)\end{array}$ & $\begin{array}{l}4,88 \\
(93)\end{array}$ & $\begin{array}{l}112 \\
(65)\end{array}$ \\
\hline ICSR - 29 & $\begin{array}{l}1,90 \\
(94)\end{array}$ & $\begin{array}{c}21,23 \\
(99)\end{array}$ & $\begin{array}{l}7,47 \\
(87)\end{array}$ & $\begin{array}{l}62,33 \\
(100)\end{array}$ & $\begin{array}{c}46,17 \\
(97)\end{array}$ & $\begin{array}{c}5,86 \\
(112)\end{array}$ & $\begin{array}{l}142 \\
(82)\end{array}$ \\
\hline INTA-CNIA (T) & $\begin{array}{c}2,02 \\
(100)\end{array}$ & $\begin{array}{l}21,35 \\
(100)\end{array}$ & $\begin{array}{c}8,57 \\
(100)\end{array}$ & $\begin{array}{l}62,26 \\
(100)\end{array}$ & $\begin{array}{l}47,62 \\
(100)\end{array}$ & $\begin{array}{c}5,22 \\
(100)\end{array}$ & $\begin{array}{c}173 \\
(100)\end{array}$ \\
\hline DMS $(0,05 \%)$ & 1203,5 & 11,86 & 1,23 & 2,26 & 2,25 & & \\
\hline
\end{tabular}

Los valores entre paréntesis indican el porcentaje sobre la variedad testigo INTA-CNIA.

RSH: rendimiento seco de hoja, RFR: rendimiento fresco de rastrojo, MAT: materia proteica total, NDF: fibra detergente, neutro, SMS: digestibilidad enzimática in vitro de la materia seca, ADL/NDF: proporción de lignina en fibras totales, MPB h: materia proteica bruta en hojas.

insumos en países en desarrollo (White et al. 1981, Capper 1988, Rattunde 1998).

\section{Identificación de genotipos promisorios para uso de doble propósito}

En los análisis de rendimiento y calidad de hojas, los genotipos V 144, V 142 e ICSR 89064 presentaron los mayores valores de MAT, superando en 16, 4 y $1 \%$, respectivamente, a la variedad testigo INTA-CNIA; los más bajos porcentajes de ADL/NDF y NDF, y por consiguiente, los mayores valores de SMS. Sureño, SH 688 y V 142 sobresalieron en MPBh con valores de 259,226 y $199 \mathrm{~kg} / \mathrm{ha}$, y en RFR con 55,48; 48,71 y $37,16 \mathrm{t} / \mathrm{ha}$, respectivamente, superiores al testigo INTA-CNIA (Cuadro 3).
En los análisis de rendimiento y calidad de tallos, 96-2/46-1K-1K-1K y V 142 mostraron contenidos de MAT y IVNDFD similares al testigo (3,5 y 9,23\%, respectivamente). En RSR sobresalieron BF 95-11/195, BF 95-11/160, V 144 y Sureño, con 9,48; 8,37; 7,92 y 7,62 t/ha, estos mismos genotipos obtuvieron excelente rendimiento de $\mathrm{dMO} / \mathrm{ha}$ con 323,$7 ; 326,2 ; 289,9$ y $257,6 \mathrm{~kg} / \mathrm{ha}$, respectivamente, los cuales combinaron excelente materia proteica bruta por hectárea (MPB/ha), a excepción de BF 95-11/160, superando al testigo (119 $\mathrm{kg} / \mathrm{ha}$ ) y a los demás genotipos en estudio (Cuadro 4).

Los genotipos BF 95-11/195 y BF 95-11/160, presentaron los mayores contenidos de grados Brix,con valores de 11,4 y $9,4 \%$, indicando de que estos genotipos son de buena palatabilidad en la alimentación animal. 
Cuadro 4. Calidad nutricional (tallos) de los mejores genotipos de sorgo de doble propósito. Centro Nacional de Investigación Agropecuaria (CNIA), Managua, Nicaragua. 2004.

\begin{tabular}{|c|c|c|c|c|c|c|c|c|}
\hline Variedades & RSR t/ha & H/RS & MAT & NDF & $\begin{array}{c}\text { ADL/ } \\
\text { NDF }\end{array}$ & IVNDFD & $\begin{array}{c}\text { MPB t } \\
\text { kg/ha }\end{array}$ & $\begin{array}{c}\text { dMO t } \\
\mathrm{kg} / \mathrm{ha}\end{array}$ \\
\hline Sureño & $\begin{array}{c}7,62 \\
(139)\end{array}$ & $\begin{array}{l}0,30 \\
(80)\end{array}$ & $\begin{array}{l}2,83 \\
(81)\end{array}$ & $\begin{array}{l}67,70 \\
(103)\end{array}$ & $\begin{array}{c}8,29 \\
(121)\end{array}$ & $\begin{array}{l}8,36 \\
(91)\end{array}$ & $\begin{array}{c}158 \\
(133)\end{array}$ & $\begin{array}{l}257,6 \\
(161)\end{array}$ \\
\hline V144 & $\begin{array}{c}7,92 \\
(144)\end{array}$ & $\begin{array}{l}0,25 \\
(66)\end{array}$ & $\begin{array}{l}2,52 \\
(72)\end{array}$ & $\begin{array}{c}60,92 \\
(93)\end{array}$ & $\begin{array}{c}9,10 \\
(133)\end{array}$ & $\begin{array}{l}6,47 \\
(70)\end{array}$ & $\begin{array}{c}149 \\
(125)\end{array}$ & $\begin{array}{l}289,9 \\
(181)\end{array}$ \\
\hline V142 & $\begin{array}{l}5,00 \\
(91)\end{array}$ & $\begin{array}{c}0,3 \\
(80)\end{array}$ & $\begin{array}{l}3,39 \\
(97)\end{array}$ & $\begin{array}{c}64,27 \\
(98)\end{array}$ & $\begin{array}{c}8,18 \\
(119)\end{array}$ & $\begin{array}{l}9,17 \\
(99)\end{array}$ & $\begin{array}{c}119 \\
(100)\end{array}$ & $\begin{array}{l}166,4 \\
(104)\end{array}$ \\
\hline SH 688 & $\begin{array}{c}7,39 \\
(135)\end{array}$ & $\begin{array}{l}0,27 \\
(71)\end{array}$ & $\begin{array}{l}2,58 \\
(74)\end{array}$ & $\begin{array}{c}64,42 \\
(99)\end{array}$ & $\begin{array}{c}7,25 \\
(106)\end{array}$ & $\begin{array}{l}6,18 \\
(67)\end{array}$ & $\begin{array}{c}139 \\
(117)\end{array}$ & $\begin{array}{l}253,8 \\
(159)\end{array}$ \\
\hline ICSR 89064 & $\begin{array}{c}6,69 \\
(122)\end{array}$ & $\begin{array}{l}0,33 \\
(87)\end{array}$ & $\begin{array}{l}3,05 \\
(87)\end{array}$ & $\begin{array}{l}65,67 \\
(101)\end{array}$ & $\begin{array}{c}9,16 \\
(134)\end{array}$ & $\begin{array}{l}5,59 \\
(61)\end{array}$ & $\begin{array}{c}137 \\
(115)\end{array}$ & $\begin{array}{l}206,3 \\
(129)\end{array}$ \\
\hline BF 95-11/195 & $\begin{array}{c}9,48 \\
(172)\end{array}$ & $\begin{array}{l}0,19 \\
(50)\end{array}$ & $\begin{array}{l}1,82 \\
(52)\end{array}$ & $\begin{array}{l}72,73 \\
(111)\end{array}$ & $\begin{array}{c}8,46 \\
(124)\end{array}$ & $\begin{array}{l}3,87 \\
(42)\end{array}$ & $\begin{array}{c}140 \\
(118)\end{array}$ & $\begin{array}{l}323,7 \\
(202)\end{array}$ \\
\hline BF 95-11/160 & $\begin{array}{c}8,37 \\
(152)\end{array}$ & $\begin{array}{l}0,17 \\
(45)\end{array}$ & $\begin{array}{l}1,78 \\
(51)\end{array}$ & $\begin{array}{c}63,86 \\
(98)\end{array}$ & $\begin{array}{c}9,89 \\
(144)\end{array}$ & $\begin{array}{l}3,90 \\
(42)\end{array}$ & $\begin{array}{c}123 \\
(103)\end{array}$ & $\begin{array}{l}326,2 \\
(204)\end{array}$ \\
\hline ICSR - 29 & $\begin{array}{c}6,19 \\
(113)\end{array}$ & $\begin{array}{l}0,35 \\
(92)\end{array}$ & $\begin{array}{l}3,19 \\
(91)\end{array}$ & $\begin{array}{l}63,0 \\
(97)\end{array}$ & $\begin{array}{c}7,81 \\
(114)\end{array}$ & $\begin{array}{l}8,90 \\
(96)\end{array}$ & $\begin{array}{c}128 \\
(108)\end{array}$ & $\begin{array}{l}195,7 \\
(122)\end{array}$ \\
\hline BF $94-6 / 46 \mathrm{~K}-1 \mathrm{~K}-1 \mathrm{~K}-1 \mathrm{~F}$ & $\begin{array}{c}6,84 \\
(124)\end{array}$ & $\begin{array}{l}0,29 \\
(76)\end{array}$ & $\begin{array}{l}2,58 \\
(74)\end{array}$ & $\begin{array}{l}68,26 \\
(105)\end{array}$ & $\begin{array}{c}7,64 \\
(112)\end{array}$ & $\begin{array}{l}6,90 \\
(75)\end{array}$ & $\begin{array}{c}126 \\
(106)\end{array}$ & $\begin{array}{l}221,0 \\
(138)\end{array}$ \\
\hline BF $96-2 / 46-1 \mathrm{~K}-1 \mathrm{~K}-1 \mathrm{~K}$ & $\begin{array}{l}4,84 \\
(88)\end{array}$ & $\begin{array}{l}0,35 \\
(92)\end{array}$ & $\begin{array}{c}3,56 \\
(102)\end{array}$ & $\begin{array}{l}66,98 \\
(103)\end{array}$ & $\begin{array}{c}7,14 \\
(104)\end{array}$ & $\begin{array}{l}8,32 \\
(90)\end{array}$ & $\begin{array}{l}110 \\
(92)\end{array}$ & $\begin{array}{c}142,4 \\
(89)\end{array}$ \\
\hline INTA-CNIA(T) & $\begin{array}{c}5,50 \\
(100)\end{array}$ & $\begin{array}{c}0,38 \\
(100)\end{array}$ & $\begin{array}{c}3,50 \\
(100)\end{array}$ & $\begin{array}{l}65,25 \\
(100)\end{array}$ & $\begin{array}{c}6,85 \\
(100)\end{array}$ & $\begin{array}{c}9,23 \\
(100)\end{array}$ & $\begin{array}{c}119 \\
(100)\end{array}$ & $\begin{array}{l}160,0 \\
(100)\end{array}$ \\
\hline DMS $(0,05 \%)$ & 1,49 & 0,12 & 0,94 & 6,70 & 1,40 & 3,06 & & \\
\hline
\end{tabular}

Los valores entre paréntesis indican el porcentaje sobre la variedad testigo INTA-CNIA.

RSR: rendimiento seco de rastrojo, H/RS: relación hoja y rendimiento seco, MAT: materia proteica total, NDF: fibra detergente neutro, ADL/NDF: proporción de lignina en fibras totales, IVDNDF: digestibilidad de la fibra detergente neutro, MPB t: materia proteica bruta en tallos, dMO t: digestibilidad in vivo de la materia orgánica en tallos.

El mayor potencial de rendimiento de grano lo presentaron SH 688, BF 95-11/195, V 142 y Sureño, superando al testigo (5080 kg/ha) en $26,19,11$ y $8 \%$, respectivamente, estos mismos genotipos sobresalieron en RFR, a excepción de V 142 (Cuadro 5).

Los mejores genotipos que se han destacado por su alto rendimiento (grano y rastrojo) y calidad nutricional, como son BF 95-11/195, Sureño, SH 688, V 142 y V 144, son de fácil acceso para los pequeños y medianos productores, podrán seleccionar su propia semilla para próximas siembras por ser variedades de polinización libre y por consiguiente su semilla es accesible debido a su bajo costo en comparación con la semilla híbrida.

Sureño superó a todos los genotipos en MPB en hojas y tallos, aceptable dMO y rendimiento de grano. Las variables IVNDFD y ADL/NDF de los tallos, presentaron valores de heredabilidad superior a 0.4 , siendo importante para mejorar la calidad nutricional del rastrojo de sorgo.

Las características de calidad nutritiva del rastrojo y productividad de los genotipos señalados se 
Cuadro 5. Síntesis de las características agronómicas de los mejores genotipos de sorgo de doble propósito evaluados en los años 2003, 2004 y 2005. Centro Nacional de Investigación Agropecuaria (CNIA), Managua, Nicaragua.

\begin{tabular}{|c|c|c|c|c|c|c|}
\hline Variedades & $\mathbf{N} !$ & $\begin{array}{c}\text { Días a } \\
\text { floración }\end{array}$ & $\begin{array}{c}\text { Altura de } \\
\text { planta }(\mathrm{cm})\end{array}$ & $\begin{array}{c}\text { BRIX } \\
\%\end{array}$ & $\begin{array}{l}\text { RGR } \\
\mathrm{kg} / \mathrm{ha}\end{array}$ & $\begin{array}{c}\text { RFR } \\
\text { t/ha }\end{array}$ \\
\hline Sureño & 3 & 70 & 253 & $\begin{array}{c}8,6 \\
(132)\end{array}$ & $\begin{array}{l}5465 \\
(108)\end{array}$ & $\begin{array}{l}43,77 \\
(166)\end{array}$ \\
\hline V144 & 3 & 66 & 224 & $\begin{array}{c}8,6 \\
(132)\end{array}$ & $\begin{array}{c}5000 \\
(98)\end{array}$ & $\begin{array}{l}27,80 \\
(105)\end{array}$ \\
\hline V142 & 2 & 66 & 230 & $\begin{array}{c}7,5 \\
(122)\end{array}$ & $\begin{array}{l}5647 \\
(111)\end{array}$ & $\begin{array}{l}27,92 \\
(106)\end{array}$ \\
\hline SH 688 & 2 & 55 & 230 & $\begin{array}{c}6,6 \\
(107)\end{array}$ & $\begin{array}{l}6401 \\
(126)\end{array}$ & $\begin{array}{l}40,00 \\
(151)\end{array}$ \\
\hline ICSR 89064 & 3 & 68 & 166 & $\begin{array}{l}6,3 \\
(97)\end{array}$ & $\begin{array}{c}4690 \\
(92)\end{array}$ & $\begin{array}{l}28,44 \\
(108)\end{array}$ \\
\hline BF 95-11/195 & 1 & 57 & 277 & $\begin{array}{c}9,4 \\
(145)\end{array}$ & $\begin{array}{l}6071 \\
(119)\end{array}$ & $\begin{array}{l}31,24 \\
(118)\end{array}$ \\
\hline BF 95-11/160 & 1 & 58 & 265 & $\begin{array}{c}11,4 \\
(175)\end{array}$ & $\begin{array}{c}4990 \\
(78)\end{array}$ & $\begin{array}{l}27,51 \\
(104)\end{array}$ \\
\hline ICSR - 29 & 3 & 66 & 157 & $\begin{array}{c}6,9 \\
(106)\end{array}$ & $\begin{array}{r}4130 \\
(81)\end{array}$ & $\begin{array}{l}21,05 \\
(80)\end{array}$ \\
\hline BF $94-6 / 46 K-1 K-1 K-1 F$ & 2 & 54 & 169 & $\begin{array}{c}9,0 \\
(138)\end{array}$ & $\begin{array}{c}4168 \\
(83)\end{array}$ & $\begin{array}{c}22,01 \\
(76)\end{array}$ \\
\hline INTA-CNIA (T) & 3 & 67 & 164 & $\begin{array}{c}6,5 \\
(100)\end{array}$ & $\begin{array}{l}5080 \\
(100)\end{array}$ & $\begin{array}{l}26,42 \\
(100)\end{array}$ \\
\hline
\end{tabular}

$\mathrm{N}$ !: número de años de evaluación para las variables agronómicas.

Los valores entre paréntesis indican el porcentaje sobre la variedad testigo INTA-CNIA en evaluaciones comunes.

BRIX: grados Brix, RGR: rendimiento de grano, RFR: rendimiento fresco de rastrojo.

podrían utilizar en los programas de fitomejoramiento; desarrollando nuevas variedades y líneas, utilizando los mejores genotipos identificados en el presente estudio como restauradores y progenitores para formar híbridos.

\section{LITERATURA CITADA}

Amador, AL; Boschini, C. 2000. Calidad nutricional de la planta de sorgo negro forrajero (Sorghum almum) para alimentación animal. Agronomía Mesoamericana 11(2):79-84.

Badve, VC; Nisal, PR; Joshi, AL; Rangnekar, DV. 1994. Genotype and environment effects on sorghum stover production and quality. In Joshi, AL; Doyle, PT; Oosting, SJ. eds. Variation in the quantity and quality of fibrous crop residues. Proceedings of the National
Seminar held at the BAIF Development Research Foundation. Pune, Mahaiarashtra, India. p. 9-19.

Bartle, SJ; Klopfenstein, TJ. 1988. Non-chemical opportunities for improving crop residue feed quality: a review. J. Prod. Agric. 1:356-361.

Barriere, Y; Thomas, J; Denoue, D. 2008. QTL mapping for lignin content, lignin monomeric composition, p-hydroxycinnamate content and cell wall digestibility in the maize recombinant inbred line progeny F838 x F286. Plant Science 175:585-595.

Bastianelli, D; Hervouet, C. 2000. Techniques d'analyses des aliments pour animaux. CIRAD - EMVT. Montpellier, Francia. 70 p.

Bolleta, A; Vallati, A. 2006. Evaluación de sorgos diferidos: producción de materia seca y calidad. INTA EEA, Campaña 2006/2007. 7 p. (en línea). Consultado 10 ago. 2009. Disponible en http://www.produccionanimal.com.ar/produccion 
Boschini, C; Amador, A. 2000. Degradabilidad ruminal de la planta de sorgo negro forrajero (Sorghum almum) en diferentes etapas de crecimiento. Agronomía Mesoamericana 12(2):169-174.

Boschini, C; Elizondo, J. 2005. Determinación de la calidad y la producción de sorgo negro forrajero (Sorghum almum) en edades para ensilar. Agronomía Mesoamericana 16(1):29-36.

Bramel-Cox, PJ; Kumar, KA; Hancock, JD; Andrews, DJ. 1995. Sorghum and millets for forage and feed. In Dendy, DAV. ed. Sorghum and millets chemistry and technology. American Assoc. Cereal Chemists, Inc. St. Paul. p. 325-364.

Capper, BS. 1988. Genetic variation in the feeding value of cereal straw. Anim. Feed Sci. Technol. 21:127-140.

Caravetta, GJ; Cherney, JH; Johnson, KD. 1990. Within-row spacing influences on diverse sorghum genotypes. II. Dry matter yield and forage quality. Agronomy Journal 82:210-215.

Colin-Schoellen, O; Jurjanz, S; Laurent, F. 2000. Metabolizable protein supply (PDIE) and restricted level of ruminally degradable nitrogen (PDIN) in total mixed rations: effect on milk production and composition and on nitrogen utilization by dairy cows. Livestock Production Science 67(1):41-53. (en línea). Consultado 27 julio 2009. Disponible en http://www.ingentaconnect.com/content/els

Corrales, JC. 1986. Efecto de la densidad de siembra y la fertilización nitrogenada sobre la producción de biomasa y la calidad de sorgo negro forrajero (Sorghum almum). Tesis de Lic. en Ingeniería Agronómica con énfasis en Zootecnia. Escuela de Zootecnia. Universidad de Costa Rica, San José, Costa Rica. 67 p.

Dahlberg, J; Berenji, J; Sikora, V; Latkovic, D. 2011. Assessing sorghum [Sorghum bicolor (L) Moench] germplasm for new traits: food, fuels \& unique uses. Maydica 56:85-92.

Fales, S. 1986. Effects of temperature on fiber concentration, composition and in vitro digestion kinetics of tall fescue. Agronomy Journal 78:963-966.

FAO (Food and Agriculture Organization). 1995. El sorgo y el mijo en la nutrición humana. Colección FAO: Alimentación y nutrición. Depósitos de Documentos de la FAO. Roma - Italia No 27. 197 p. (en línea). Consultado 12 oct. 2012. Disponible en http://www.fao.org

García, GJ. 1981. Cultivos herbáceos. Agrociencia. Barcelona, España. 534 p.

García, RO; García, OD. 2007. Evaluación del efecto de diferentes niveles de amonificación sobre la calidad nutritiva del rastrojo de sorgo. Tesis de ingeniero agrónomo. Universidad Nacional Agraria (UNA), Managua, Nicaragua. 34 p. (en línea). Consultado 7 nov. 2009. Disponible en http://cenida.una.edu.ni/ tesis/tnp34p216e.pdf

Gupta, SC; Paliwal, RL; Nanda, JS. 1976. Combining ability for forage yield and quality characters in sorghum (Sorghum bicolor L.) Moench. Egypt J. Gent. Cytol. 5:89-97.

Hanson, WD. 1963. Heritability. In Hanson, WD; Robinson, HF. eds. Stat. Genetics and Plant Breeding NASNRC. p. 125-140 (en línea). Consultado 12 mayo 2010. Disponible en http//www.redpav.avepagro.org. ve/fagro/v24

Hemanth, KM; Yellamanda, RT; Sreenivas, B. 1998. Phenotypic stability for grain equivalent in dual-purpose sorghum (Sorghum bicolor). Agricultural Research Station, Acharya N. G. Ranga Agricultural University, Andhra Pradesh, Anantapur 515 001. Indian Journal of Agricultural Sciences 69(7):494-496.

INETER (Instituto Nicaragüiense de Estudios Territoriales). 2004. Dirección General de Meteorología. Resumen meteorológico diario. Estación las Mercedes. Managua, Nicaragua (en línea). Consultado 12 ago. 2009. Disponible en http//www.ineter.gob.ni

ILRI (International Livestock Research Institute). 2012. Genetic enhancement of sorghum and millet residues fed to ruminants. ILRI Impact Assessment Series 3, Nairobi, Kenia. 52 p. (en línea). Consultado 14 nov. 2012. Disponible en http://www.ilri.org/InfoServ/Webpub/fulldocs/impact3/chapter2.htm.

Kelly, TG; Rao, PP. 1993. Sorghum and millet in Asia. In Byth, DE. ed. Sorghum and millets commodity and research environments. ICRISAT, Patancheru, Andhra Pradesh, India. p. 95-117.

Kristjanson, PM; Zerbini, E. 1999. Genetic enhancement of sorghum and millet residues fed to ruminants: an ex ante assessment of returns to research. ILRI Impact Assessment Series 3. International Livestock Research Institute. Kenya. 44 p.

Lodhi, GP; Dangi, OP. 1981. Genetics of yield and quality characters in forage sorghum. Forage Res. 7:57-71.

MAG (Ministerio de Agricultura y Ganadería). 1971. Manual práctico para interpretación de suelos. Catastro e Inventario de Recursos Naturales. CENIDA, Universidad Nacional Agraria. Managua, Nicaragua. 39 p.

MAGFOR (Ministerio Agropecuario y Forestal). 2009. Consolidado de granos básicos. Ciclo agrícola 2008/2009. Departamento de Estadísticas Continuas 
de la Dirección de Estadísticas. Managua, Nicaragua (en línea). Consultado 25 julio 2010. Disponible en http//www.magfor.gob.ni

McDowell, LR. 1974. Tablas de composición de alimentos de América Latina. Gainesville, Florida. USA. 21 p.

Mieres, J; Banchero, G; Tieri, M; La Mana, A. 2011. En tiempos de la agricultura: ¿Cómo mejorar la utilización del rastrojo de sorgo para la alimentación de ganado bovino?. La Estanzuela, INIA, Serie Actividades de Difusión No. 658 (en línea). Consultado 19 noviembre 2012. Disponible en http://www.produccion-animal.com.ar/produccion_y_manejo_pasturas/ pastoreo\%20sistemas/55-es_conveniente_el_encierre_nocturno.pdf.

Murray, SC; Sharma, A; Rooney, WL; Klein, PE; Mullet, JE; Mitchell, SE; Kresovich, S. 2008. Genetic improvement of sorghum as a biofuel feedstock: I. QTL for Stem sugar and grain nonstructural carbohydrates. Cornell Univ. Ithaca, NY. Crop Science 48:2165-2179.

Nuñez, HG; Cantú, BJ. 2001. Producción, composición química y digestibilidad del forraje de sorgo x sudan de nervadura café en la región norte de México. Facultad de Agronomía y Zootecnia. División de Estudios y Postgrados (en línea). Consultado 7 mayo 2010. Disponible en http//www.tecnicapecuaria.org.mx

Parthasarathy, P; Hall, AJ. 2003. Importance of crop residues in crop livestock systems in India and farmers perceptions of fodder quality in coarse cereals. Patancheru, Andhra Pradesh, India. Field Crops Research 84:189-198.

Pastora, J; Téllez, O. 2004. Evaluación de híbridos de sorgo (Sorghum bicolor L. Moench) para forraje. Informe Técnico. INTA-Pacífico Norte. Nicaragua. 9 p.

Rattunde, HFW. 1998. Early - maturing dual - purpose sorghums: Agronomic trait variation and covariation among landraces. Plant Breeding 117:33-36.

Rattunde, HFW; Zerbini, E; Chandra, S; Flower, DJ. 2001. Stover quality of dual - purpose sorghums: genetic and environmental sources of variation. Field Crops Research 71:1-8.

Resende, MDV. 2002. Genética biométrica y estadística no melhoramento de plantas perennes. Brasilia: EMBRAPA. Información tecnológica (en línea). Consultado 12 mayo 2010. Disponible en http//www.ufsm.br/cienciaforestal/artigos/v16n2
Ross, WM; Gorz, HJ; Haskins, FA; Hookstra, GH; Rutto, JK; Ritter, R. 1983. Combining ability effects for forage residue traits in grain sorghum hybrids. Crop Science 23:97-101.

Sanford, SG. 1989. Crop residues/livestock relationships. In Renard, C; Van Den Beldt, RJ; Parr, JF. eds. Soil, crop, and water management systems for rainfed agriculture in the Sudano - Sahelian zone. Proc. Int. Workshop. Niamey, Niger. ICRISAT. Patancheru, India. p. 169182.

Shenk, JS; Elliot, FC. 1971. Plant compositional changes resulting from two cycles of directional selection for nutritive value of alfalfa. Crop Science 11:521-524.

Trouche, G; Hocde, H; Aguirre, S; Martínez, F; Gutiérrez, N. 2006. Dinámicas campesinas y fitomejoramiento participativo: El caso de los sorgos blancos (Sorghum bicolor L. Moench) en la región norte de Nicaragua. Agronomía Mesoamericana 17(3):407-425.

Van Soest, PJ; Wine, RH. 1967. Use of detergents in the analysis of fibrous feeds. IV. Determination of plant cell walls constituens. J. Assoc. Off Anal Chem. 50:50-55.

Vargas, RC. 2005. Valoración nutricional y degradabilidad ruminal de genotipos de sorgo forrajero (Sorghum sp). Agronomía Mesoamericana 16(2):215-223.

Villegas, O. 1990. Producción y valor nutricional de sorgo de sorgos forrajeros y sus ensilados a diferentes edades de cosecha. Tesis Ing. Agrónomo. Sede Regional de Guanacaste. Universidad de Costa Rica. Liberia. Costa Rica. 91 p.

Vogel, KP; Haskins, FA; Gorz, HJ. 1981. Divergent selection for in vitro dry matter digestibility in switchgrass. Crop Science 21:39-41.

White, LM; Hartman, GP; Bergman, JW. 1981. In vitro digestibility, crude protein and phosphorus content of straw of winter wheat, spring wheat, barley and oat cultivars in eastern Montana. Agronomy Journal 73: 117-121.

Youngquist, JB; Carter, DC; Clegg, MD. 1990. Grain and forage yield and stover quality of sorghum and millet in low rainfall environments. Exp. Agric. 26:279-286.

Zerbini, E; Thomas, D. 2003. Opportunities for improvement of nutritive value in sorghum and pearl millet residues in South Asia through genetic enhancement. Field Crops Research 84:3-15. 
